\title{
Perfil de lípidos en recién nacidos sanos y su correlación con los niveles de lípidos maternos
}

Irina E. Juárez, M.J.S., ${ }^{(1)}$ Gerardo Rivera-Silva, M.B., (2) Juan M. Mejía-A rangure, M. en C., (3) José A. Mercado-A rellano, M.J.S., ${ }^{(4)}$ Silvia Díaz-Bensussen, Q .F.B., J.S. ${ }^{(5)}$

\author{
Juárez IE, Rivera-Silva G, Mejía-Arangure JM, \\ Mercado-A rellano JA, Díaz-Bensussen S. \\ Perfil de lípidos en recién nacidos sanos \\ y su correlación con los niveles \\ de lípidos maternos. \\ Salud Publica Mex 1999;41:405-409.
}

\section{Resumen}

Objetivo. Determinar los niveles séricos de lípidos, colesterol, triglicéridos y lipoproteínas de un grupo de recién nacidos y sus madres, y conocer si existe correlación entre los valores de ambas poblaciones. Material y métodos. Se tomaron muestras de sangre de 200 recién nacidos sanos, así como de sus madres, las muestras fueron procesadas por el autoanalizador. Los datos se analizaron en el programa Epi Info 6. Se obtuvieron mediciones de tendencia central, y sus dispersiones análisis de varianza y coeficiente de correlación de Pearson. Resultados. Los niveles promedio de lípidos totales en los recién nacidos fueron de $625.2 \pm 130$ $\mathrm{mg} / \mathrm{dl}$; de colesterol $158.8 \pm 44 \mathrm{mg} / \mathrm{dl}$, triglicéridos $136.9 \pm 97$ $\mathrm{mg} / \mathrm{dl}$, LDL $70.5 \pm 23.9 \mathrm{mg} / \mathrm{dl}, \mathrm{HDL} 52.7 \pm 14 \mathrm{mg} / \mathrm{dl}$, VLDL $26.5 \pm 15.5 \mathrm{mg} / \mathrm{dl}$. Conclusiones. Los niveles de colesterol en los recién nacidos fueron semejantes a los encontrados por Alpers y superiores a los reportados por el Comité Americano de Bioquímica Pediátrica en Estados Unidos de América (EUA). Se encontró discreta asociación entre los niveles maternos y de sus recién nacidos.

Palabras clave: lípidos; recién nacido; madres; México

\author{
Juárez IE, Rivera-Silva G, Mejía-A rangure JM, \\ Mercado-A rellano JA, Díaz-Bensussen S. \\ Lipid profile \\ in healthy newborns \\ and their mothers. \\ Salud Publica Mex 1999;41:405-409.
}

\begin{abstract}
A bstract
Objective.To determine serum levels of lipid, cholesterol, triglycerides and lipoproteins in newborns and their mothers and to know the association between them. Material and methods Blood samples were taken from 200 newborns and from their mothers, and analyzed for lipid, cholesterol, triglyceride and lipoprotein content in an autoanalyzer. D ata were analyzed with the Epi Info 6 program. Central tendency measurements, variance analysis and Pearson correlation co efficient were obtained. Results Average lipid levels in newborn blood samples was 625.2 130 , $158.8 \pm 44 \mathrm{mg} / \mathrm{dl}$ of cholesterol, $136.9 \pm 97 \mathrm{mg} / \mathrm{dl}$ of triglycerides, $70.5 \pm 23.9 \mathrm{mg} / \mathrm{dl} \mathrm{LDL}, 52.7 \pm 14 \mathrm{mg} / \mathrm{dl} \mathrm{HDL}$ and $26.5 \pm 15.5 \mathrm{mg} / \mathrm{dl}$ VLDL. Conclusions N ewborn cholesterol levels were similar to values reported by Alpers and higher than levels published by the American Committee of Pediatric Biochemistry in USA. An association between levels in mothers and their children was found.
\end{abstract}

Key words: lipids; newborn; mothers; Mexico

(1) Jefe de Departamento Clínico, Pediatría Médica Prescolares, Hospital de Pediatría, Centro Médico N acional Siglo XXI, Instituto Mexicano del Seguro Social (IMSS). México.

(2) Departamento de Pediatría Médica Prescolares, Hospital de Pediatría, Centro Médico N acional Siglo XXI, IMSS, México.

(3) Unidad de Investigación en Epidemiología Clínica, Hospital de Pediatría, Centro Médico N acional, Siglo XXI, IMSS, México.

(4) Jefe de Departamento Clínico en Educación Médica e Investigación, Hospital de Pediatría, Centro Médico N acional Siglo XXI, IMSS.

(5) Jefe del Departamento de Bioquímica, Hospital de Pediatría, Centro Médico N acional Siglo XXI, IMSS, México.

Fecha de recibido: 12 de junio de 1998 - Fecha de aprobado: 4 de junio de 1999

Solicitud de sobretiros: Dra. Irina E. Juárez Muñoz, o Dr. J.A gustín Mercado A rellano.A mores 620, colonia del Valle, 03100 México, D.F., México.

Correo electrónico: agustínmercado@ hotmail.com 
$\mathrm{E}$ 1 colesterol y otros lípidos como los cerebrósidos y los gangliósidos constituyen entre 70 y $75 \%$ del peso seco de la mielina en los fetos humanos en vías de maduración. ${ }^{1}$ En el tejido cerebral aún inmaduro, específicamente en las células de la glía, se observan pequeñas gotas de ésteres de colesterol; además, los lípidos son importantes componentes del cerebro humano, tanto en su funcionamiento como en su estructura. Durante la vida fetal, el colesterol tiene la importante función, entre otras, de ser el sustrato para la génesis de los esteroides y ácidos biliares. Aunado a ello, los ácidos grasos y el colesterol son las únicas fracciones de lípidos presentes en la circulación materna que cruzan la placenta por difusión simple, y se ha descrito que las concentraciones maternas y fetales de estos lípidos correlacionan estrechamente. ${ }^{2,3}$

Los niveles séricos de colesterol dependen de la interacción entre influencias ambientales y genéticas. ${ }^{4,5}$ Al respecto, el estudio Bogalusa (Bogalusa Heart Study $)^{6}$ estableció, en edades pediátricas, la relación directa de factores ambientales -como la ingesta excesiva de carbohidratos refinados, proteínas animales, grasa animal y sodio- con la presencia de enfermedades cardiovasculares asociadas a lesiones ateromatosas ocasionadas principalmente por niveles anormales de colesterol. ${ }^{7}$ La influencia de los aspectos genéticos se ha determinado a partir de observaciones hechas entre los descendientes de padres con problemas isquémicos coronarios, quienes tienen un mayor riesgo de ser portadores de alteraciones en las lipoproteínas. ${ }^{8-10}$

En Estados Unidos de América (EUA) y algunos países europeos se han efectuado mediciones séricas de colesterol en varones de entre 5 y 9 años de edad y, aun cuando los hábitos dietéticos y patrones de herencia varían, se han obtenido valores promedio similares; sin embargo, las repercusiones a largo plazo, medidas a partir de la mortalidad atribuida a enfermedad cardiovascular en varones de entre 33 y 49 años de edad, difieren de un país a otro debido, probablemente, a diversos factores ambientales. ${ }^{11,12}$

En México se han realizado estudios ${ }^{9}$ en grupos de edad de entre 5 y 18 años, todos ellos en comunidades rurales e indígenas; al comparar las cifras de colesterol observadas en estos estudios con las obtenidas en comunidades de EUA, se encontró que las primeras eran marcadamente inferiores. El nivel socioeconómico parece tener influencia directa sobre las cifras de lípidos, como lo muestra Cueto, ${ }^{13}$ pues son significativamente más altas en los niños mexicanos cuyas familias tienen un mayor nivel de ingreso. En otro estudio nacional se determinaron los niveles séricos entre los diferentes grupos de edad, pero no se incluyó a los recién nacidos. ${ }^{14}$

Los estudios elaborados en países anglosajones han mostrado que los niveles de fosfolípidos, triglicéridos y colesterol séricos en neonatos están asociados con los niveles circulantes en la madre; esta relación incluso se sigue presentando en la etapa perinatal, aunque con cifras menores. ${ }^{1,15}$ En fecha reciente Sánchez y colaboradores publicaron un estudio en el que analizaron los niveles de colesterol de 548 recién nacidos españoles sanos, de los cuales $6 \%$ tuvieron valores superiores a los $100 \mathrm{mg} / \mathrm{dl}(>2.59 \mathrm{mmol} / \mathrm{l}) .{ }^{16} \mathrm{Sin}$ embargo, estas determinaciones fueron hechas a partir de muestras de sangre mezclada arteriovenosa de cordón, lo que puede limitar la exactitud del valor obtenido; inclusive, en estudios recientemente llevados a cabo, no se ha determinado con exactitud la relación que existe entre los lípidos de las madres y los de sus recién nacidos. ${ }^{17}$ Se consultó Medline, y en los últimos cinco años (1994-1998) no se han registrado informes con este tipo de datos; por tal motivo, el clásico estudio de Glueck de hace 25 años continúa siendo empleado como patrón de referencia. ${ }^{18}$

El presente trabajo se elaboró con el objeto de determinar los niveles de colesterol, triglicéridos y lipoproteínas en sujetos residentes de las delegaciones Xochimilco, Coyoacán e Iztapalapa de la ciudad de México.

\section{Material y métodos}

Previo se elaboró una carta de consentimiento informado y se logró la aceptación por escrito de las madres para participar en el estudio. Este se llevó a cabo por los departamentos de Ginero-obstetricia y Pediatría, Hospitales General 32 y 1A, del Instituto Mexicano del Seguro Social (IMSS), localizados al sur del Distrito Federal. La afluencia de pacientes proviene, principalmente, de las delegaciones Xochimilco, Coyoacán e Iztapalapa. El protocolo fue avalado por los comités de investigación y ética locales.

La población inicial incluyó a 200 madres sanas, con las siguientes condiciones clínicas: edad de entre 20 y 34 años, sin padecimientos asociados como la diabetes -incluyendo la gestacional-, y sin nefropatía, enfermedad cardiovascular, epilepsia, trastornos mentales o desnutrición; ${ }^{15,19}$ los datos fueron obtenidos al analizar la historia clínica general y la obstétrica. Todas las madres cursaron embarazo normal, con productos únicos obtenidos por parto eutócico vaginal, sin evidencia, al interrogatorio, de exposición a tóxicos, radiaciones ni de haber adquirido infecciones congé- 
nitas; igualmente, todas tuvieron un embarazo calificado como de bajo riesgo, y sus respectivos hijos nacieron a término (38-42 semanas de gestación), sin malformaciones congénitas visibles al examen físico, clínicamente sanos, con pesos adecuados para la edad gestacional -el límite inferior se ubicó por arriba de los $2500 \mathrm{~g}$, y el superior, por debajo de los $4000 \mathrm{~g}-{ }_{,}{ }^{15}$ sin distocia de presentación y con calificación de Apgar $\geq 8$ al minuto y a los cinco minutos del nacimiento. $15,16,19$

Previamente a la toma de las muestras, las madres cumplieron con un ayuno de entre 12 y 16 horas y reposaron de 10 a 15 minutos. A los recién nacidos se les extrajo la muestra dentro de las primeras seis horas de vida. Por punción venosa se extrajo a cada paciente $3 \mathrm{ml}$ de sangre. Las muestras fueron colectadas en tubos de ensayo sin anticoagulante; los sueros se separaron dentro de las tres horas siguientes, por medio de una centrífuga refrigerada durante 15 minutos a $3000 \mathrm{rpm}$ y, previamente identificados, se almacenaron a $4^{\circ} \mathrm{C}$ y se procesaron dentro de los primeros nueve días de su obtención. ${ }^{20}$

El colesterol y los triglicéridos se cuantificaron por métodos enzimáticos en el autoanalizador Express 550, marca Ciba Cornig, con los métodos de referencia del Centro para la Prevención y el Control de las Enfermedades, (Atlanta GA Center for Disease Control) (19829 S-N 631-008-24542 CDC). Para la medición de lipoproteínas de alta densidad (HDL-C) se precipitaron aquellas cuya densidad era menor a 1063 , y en el sobrenadante se analizó el HDL-C, por el mismo método enzimático, con el autoanalizador mencionado. ${ }^{21}$ Los lípidos totales se midieron por colorimetría empleando equipo comercial de la casa Merck. Las lipoproteínas de baja densidad (LDL-C) y de muy baja densidad (VLDL-C) se estimaron de acuerdo con la ecuación de Friedewald:22

- $\quad$ LDL-C $=$ colesterol total $-(H D L-C+V L D L-C)$

- $\quad V L D L-C=$ triglicéridos $/ 5$

La ecuación de Friedewald ha sido validada para neonatos y previamente publicada. ${ }^{14}$

El error estándar del análisis se estimó en 1.5\%, y el coeficiente de variación intraensayo osciló entre 2 y $4.2 \%$.

Si la concentración de triglicéridos y lípidos totales es $<400 \mathrm{mg} / \mathrm{dl}$, se utiliza la primera fórmula, y si el valor es $<1000 \mathrm{mg} / \mathrm{dl}$, se recurre a la segunda.

Para el análisis estadístico se utilizaron medidas de tendencia central; se calcularon medias con desviación estándar, así como medianas, y se establecieron los valores percentilares de 25,50 y 75 para cada una de las seis variables estudiadas en los recién nacidos.
Las comparaciones entre las variables se realizaron mediante análisis de varianza, y la correlación entre los valores maternos y los de sus recién nacidos se analizó mediante el coeficiente de correlación de Pearson. ${ }^{23}$

\section{Resultados}

De las 200 muestras sanguíneas obtenidas de los recién nacidos, 24 (12\%) se hemolizaron, por lo que sólo se incluyeron en el trabajo las de 176 pacientes: 95 del sexo masculino $(53.9 \%)$ y 81 del femenino $(46 \%)$. El peso promedio de los recién nacidos fue de $3277 \pm 543$ $\mathrm{g}$, y la edad gestacional, de $38.2 \pm 0.4$ semanas. La edad materna promedio fue de $22.7 \pm 4.2$ años, y el peso, de $76.5 \pm 3.9 \mathrm{~kg}$.

Con el fin de establecer una correlación entre los niveles séricos de lípidos de las madres y los de sus productos, primero se realizó una estratificación de los niveles de lípidos maternos por grupos de edad (cuadro I), con el objeto de garantizar que esta variable no influyera en los resultados, y como se aprecia en dicho cuadro, no existieron diferencias significativas entre los estratos. Posteriormente, se aplicó el coeficiente de correlación de Pearson entre los niveles maternos y los de los neonatos, con los siguientes resultados: en lípidos totales se obtuvo una $r=0.54$; para colesterol y triglicéridos, los valores de $r$ fueron de 0.03 y 0.22 , respectivamente; para HDL, la correlación fue moderada $(r=0.49)$, y para LDL y VLDL, los valores de $r$ fueron de 0.40 y 0.45 , respectivamente (cuadro II).

\section{Discusión}

Los niveles promedio de colesterol obtenidos en los recién nacidos del presente estudio fueron de

\section{Cuadro I \\ Comparación de niveles SÉRICOS de Lípidos maternos por grupos de edad. Ciudad de México, 1996}

\begin{tabular}{|c|c|c|c|c|}
\hline \multicolumn{5}{|c|}{ Edad materna (años) } \\
\hline Valores en mg/dl & $\begin{array}{c}<20 \\
n=69\end{array}$ & $\begin{array}{c}20-30 \\
n=103\end{array}$ & $\begin{array}{l}>30 \\
n=4\end{array}$ & $\begin{array}{l}\text { Valor } p \\
\text { (AN OVA) }\end{array}$ \\
\hline Lípidos totales & $628.2 \pm 127.3$ & $620.8 \pm 132$ & $685 \pm 146$ & 0.61 \\
\hline Colesterol total & $154.9 \pm 36$ & $157.1 \pm 47$ & $184.5 \pm 92$ & 0.88 \\
\hline Triglicéridos & $42.1 \pm 87.7$ & $127 \pm 66$ & $80 \pm 50$ & 0.17 \\
\hline HDL & $51.4 \pm 14.7$ & $53.3 \pm 14.7$ & $59.2 \pm 4.6$ & 0.53 \\
\hline$\overline{L D L}$ & $68.7 \pm 26.2$ & $71.6 \pm 22.2$ & $76.2 \pm 28.5$ & 0.67 \\
\hline VLDL & $28.4 \pm 17.5$ & $24.9 \pm 12$ & $15.7 \pm 10.1$ & 0.10 \\
\hline
\end{tabular}

HDL: lipoproteínas de alta densidad LDL: lipoproteínas de baja densidad VLDL: lipoproteínas de muy baja densidad 


\section{Cuadro II \\ Correlación entre los niveles séricos de los LÍPIDOS DE LAS MADRES Y LOS DE SUS RECIÉN NACIDOS. Ciudad de México, 1996}

\begin{tabular}{|c|c|c|c|}
\hline \multirow[b]{2}{*}{ Lípidos séricos } & \multicolumn{2}{|c|}{ Niveles promedio } & \multirow[b]{2}{*}{$\begin{array}{l}\text { Coeficiente de } \\
\text { correlación }(r)^{*}\end{array}$} \\
\hline & $\begin{array}{l}\text { M aternos } \\
(\mathrm{mg} / \mathrm{dl})\end{array}$ & $\begin{array}{l}\text { Recién nacidos } \\
(\mathrm{mg} / \mathrm{dl})\end{array}$ & \\
\hline Lípidos totales & $639.19 \pm 140$ & $625.2 \pm 130$ & 0.54 \\
\hline Colesterol total & $186.69 \pm 56$ & $172.67 \pm 154$ & 0.03 \\
\hline Triglicéridos & $142.20 \pm 103$ & $136.9 \pm 97.4$ & 0.22 \\
\hline$\overline{H D L}$ & $50.44 \pm 13$ & $52.75 \pm 14$ & 0.49 \\
\hline$\overline{L D L}$ & $73.61 \pm 22$ & $70.5 \quad \pm 23.9$ & 0.40 \\
\hline$\overline{V L D L}$ & $27.62 \pm 15$ & $26.5 \pm 15.5$ & 0.45 \\
\hline
\end{tabular}

* Coeficiente de correlación de Pearson

HDL: lipoproteínas de alta densidad

LDL: lipoproteínas de baja densidad

VLDL: lipoproteínas de muy baja densidad

$156.8 \pm 44.2 \mathrm{mg} / \mathrm{dl}$, muy semejantes a los encontrados por Alpers, ${ }^{24}$ y superiores a los $85 \mathrm{mg} / \mathrm{dl}$ (45-167 $\mathrm{mg} / \mathrm{dl}$ ) notificados por el Comité Americano de Bioquímica en Pediatría, en EUA, para el mismo grupo de edad y para los estándares locales. ${ }^{8,11,20}$ Se trata de resultados difíciles de interpretar, ya que en ese país se han notificado, entre los adultos, niveles sanguíneos de lípidos más elevados que los conocidos en la población adulta mexicana. ${ }^{14,25}$ Sin embargo, para el presente estudio las condiciones de toma de las muestras sanguíneas fueron homogeneizadas en detalle, tanto en el caso de las madres como en el de sus recién nacidos, a diferencia de estudios previos en los que las mediciones se hicieron utilizando sangre de vasos umbilicales, sin aclarar si las muestras se obtuvieron de arterias o de la vena umbilical, ${ }^{1}$ o bien, después de haber recibido algún tipo de alimentación; ; ello no permite saber con precisión si la medición se hizo a partir de una mezcla de lípidos maternos y fetales, o si los resultados se modificaron por la ingesta de lípidos de cualquier naturaleza.

Otra de las condiciones que dieron exactitud a las determinaciones del presente estudio, fue el ayuno estricto indicado a las madres, y una más fue que la medición sérica hecha a los recién nacidos se practicó antes de que éstos recibieran su primera toma de alimento. También se garantizó que los valores no se vieran influidos por condiciones externas, porque, entre otras cosas, se seleccionó a población sana desde el punto de vista nutricional. Las madres tuvieron un peso promedio de $76.5 \pm 3.9 \mathrm{~kg}$ previamente al parto; para ello debe tomarse en cuenta el incremento es- perado ocasionado por la presencia de un producto que llegó a término, por la placenta y por el líquido amniótico, así como el crecimiento de los depósitos de grasa en las glándulas mamarias y el aumento de dos litros en el agua extracelular y extravascular. De este modo, el estado nutricional de las madres se ubicó dentro de los límites de normalidad establecidos para las mujeres de 20 a 34 años. ${ }^{26,27}$ Por su parte, los hijos también presentaron un adecuado estado nutricional, ya que el peso y la talla correspondían a la edad gestacional; asimismo, al igual que los indicadores mencionados, el índice ponderal (Rohrer) se ubicó dentro de los percentiles de normalidad. ${ }^{19}$ Todos los neonatos fueron resultado de embarazos únicos a término, sin riesgos de estrés adicionales y con calificación de Apgar $\geq 8$, lo cual de algún modo garantiza buenas condiciones clínicas generales.

Un aspecto también relevante es el hallazgo de la modesta correlación existente entre los valores de los lípidos de las madres, en particular, y los de sus hijos, como previamente ha sido notificado por algunos autores, ${ }^{1}$ así como el hecho de que ello pueda atribuirse a diferencias fisiológicas y metabólicas perinatales y a una dispersión real de las mediciones. Los resultados obtenidos en el presente estudio pueden ser considerados, no obstante las limitaciones derivadas del tamaño muestral, como valores comparativos en recién nacidos a término en nuestro medio, ya que los valores utilizados en los textos actuales de neonatología se apoyan en referencias antiguas y no son locales. ${ }^{19,28,29}$ La utilidad de contar con valores de referencia actuales es indiscutible, especialmente para atender a aquellos neonatos que padecen de enfermedades graves, son apoyados con nutrición parenteral, y requieren de una vigilancia muy estrecha de diversas variables metabólicas como son los lípidos y, en particular, el colesterol; dichos valores son útiles, sobre todo, en aquellos casos en los que los neonatos reciben nutrición parenteral total y se encuentran en condiciones de estrés, pues la utilización de las grasas como sustrato energético se incrementa. ${ }^{26}$ Es necesario, sin embargo, que se lleven a cabo determinaciones similares para diferentes edades gestacionales, con los mismos fines, por medio de estudios poblacionales.

\section{Referencias}

1. Hamosh M. Lipid metabolism in the fetos. In Polin RA, Fox W W. Fetal and neonatal physiology. Lipid metabolism in the fetus. W B Saunders, 1992:291-299.

2. Miller M, Bachurick SP, Cloeey A. N ormal variations of plasma concentrations of lipids, lipoproteins and apolipoproteins. $\mathrm{Clin}$ Chem 1992;38:569-574. 
3. Havel R. Structure and metabolism of plasma lipoproteins. JAMA 1986;104:1129-1138.

4. Russell R. The pathogenesis of atheroesclerosis. N Engl J Med 1986;314:488-497.

5. Concensus Conference. Lowering blood cholesterol to prevent heart disease. JAMA 1985;253:2080-2086.

6. N ickas T, Farris RP, Smoak GC. D ietary factors relate to cardiovascular risk factors in early life. Bogalusa H eart Study.Arteriosclerosis 1988;8:193199.

7. A merican A cademy of Pediatrics. Committee on nutrition childhood diet an coronary heart disease. Pediatrics 1972;49:305-308.

8. A cademic of Pediatrics. Biochemical, clinical, epidemiologic data in the pediatrics age group. Relevant to the cholesterol hypothesis. Pediatrics 1986;78:349-360.

9. Svegeor T. Hyperlipidemia in children with family histories of premature coronary heart disease. Acta Paediatr Scand 1987;76:349-360.

10. Goldbourt U, Neufeld $H$. Genetic aspects of atheroesclerosis. Arteriosclerosis 1986;6: 357-377.

11. A merican Academy of Pediatrics. Committee on N utrition. Pediatrics 1983;80:75-80.

12. Baranowski T, Berenson G.Dynamic change of serum lipoprotein in children during adolescent and sexual maduration. Am J Epidemiol 1981;113:157-170.

13. Cueto L. Prevención de la ateroesclerosis en México. Aspectos pediátricos de la ateroesclerosis. México, D.F::AMPAC . 1989;131-150.

14. Sepúlveda B. Hyperlipidemia in children. Pediatrics 1990;78:349-360. 15. Bastida S, Sánchez-Muñiz FJ, C uesta C, Perea S,A ragonés A. Male and female cord blood lipoprotein profile differences throught the term period. J Perinat Med 1997;25:184-191.

16. Sánchez-Muñiz FJ, Bastida S, Perea S, C uesta C ,A ragonés A. Low density lipoprotein in neonates with high cord serum cholesterol levels. Acta Paediatr 1997;86:414-418.
17. Silliman K, Kretchmer N . Maternal obesity and body composition of the neonate. Biol N eonate 1995;68:384-393.

18. G lueck CJ, Hecman F, Schoenfeld M, Steiner P, PeareW. N eonatal family type II hyperlipoproteinemia: Cord blood cholesterol in 1800 births. Metabolism 1971;20:597-608.

19. Jasso-Gutiérrez L. N eonatología práctica. 4a. edición. México, D.F.: El Manual Moderno, 1995:73-85, 519-524.

20. Kronenberg F, Lobentz E, Koning P. Effect of sample storage on the measurement apoprotein, apolipoprotein $B$ and $A$, total and high density cholesterol and triglycerides. J Lipid Res 1992;35:1315-1328.

21. Rifai N, Russell W, MCN amara J. Measurement of low density lipoprotein cholesterol in serum:A status report. Clin Chem 1992;38:150-162.

22. Friedewald W T, Fredrickson DS. Estimation of concentration of low density lipoprotein cholesterol in plasma without use of ultracentrifuge. Clin Chem 1972;18:449-502.

23. D awson-Saunders B,Trapp RG. Bioestadística médica. México, D.F.: El Manual Moderno, 1993: 62-64, 187-214.

24.C loherty JP,StarkAR. Manual of neonatal care. 3a. edición. Boston:Little, Brown and Company, 1991: 723.

25. Kannel W, Castelli W. Cholesterol in the prediction of atheroesclerosis disease: $N$ ext perspectives bases the Frammingham study.Ann Intern Med 1979:90;85-91.

26. Spear ML in Spitzer AR. Intensive care of the fetus and neonate. St Louis MO : Mosby Year Book, 1996:834-842.

27. Casilla 0 E,Vargas LA. Cuadros de peso y talla para adultos mexicanos. Arch Invest Med 1980;1:157-179.

28. Klaus MH, Fanaroff AA. Care of the high risk neonate. 4a. edición. Philadelphia:W.B. Saunders Company, 1993:484.

29. Merenstein GB, Gardner SL. Handbook of neonatal intensive care. 3a. edición. St Louis MO : Mosby Year Book, 1993:207-223. 